\title{
A search for fast photometric variability in very low mass stars and brown dwarfs
}

\author{
Samrat Ghosh ${ }^{1 *}$ Soumen Mondal ${ }^{1}$, Ramkrishna Das $^{1}$, Santosh Joshi ${ }^{2}$, Sneh Lata ${ }^{2}$, \\ Siddhartha Biswas ${ }^{1}$
}

${ }^{1}$ S. N. Bose National Centre for Basic Sciences, Salt Lake, Kolkata 700106, India

${ }^{2}$ Aryabhatta Research Institute of Observational Sciences (ARIES), Manora Peak,

Nainital-263002, India

\begin{abstract}
We present here I-band time-series photometric studies of a few very low-mass stars (VLMs) and brown dwarfs (BDs) in the young (age of 2-3 Myr) IC348 star-forming region in the Perseus Molecular Cloud. Our main aim is to explore the fast rotating (of time scale of a few hours) BDs and VLMs in the I-band. From our preliminary results, we find significant variability in the young M5 dwarf 2MASS J03443896+3203196 with a rotation period of 16.037 hours.
\end{abstract}

Keywords: low-mass star - brown dwarfs - photometry - variability

\section{Introduction}

Very Low-Mass stars (VLMs) are main-sequence stars with a spectral type from mid-K to late-M and a mass from about $0.6 \mathrm{M}_{\odot}$ down to the brown dwarf limit of $0.075 \mathrm{M}_{\odot}$ (Allard et al. 1997), while Brown Dwarfs (BDs) occupy the gap between the least massive stars and the most massive planets with a theoretical mass range of $13-75 M_{J}$ with solar metallicity. Due to their low mass, their core pressure does not reach the threshold for sustaining hydrogen burning, instead, these substellar objects burn deuterium. Brown Dwarfs were first discovered in 1995 (Nakajima et al. 1995), and after that more than thousand Brown Dwarfs were discovered in our galaxy by ground- as well as space-based optical/IR all-sky surveys in various star-forming regions as well as in the field. Low temperatures of such L/T dwarfs allow molecules to condense and form dust clouds.

The majority of these VLMs and BDs are found to be photometrically variable with rapid rotation (Herbst et al. 2000; Rockenfeller et al. 2006; Zapatero Osorio et al. 2006; Crossfield 2014). Variability in these dwarfs is attributed due to the presence of surface features like magnetic spots (due to strong magnetic fields) or dust clouds, which cause optical modulation as these objects rotate, and it is possible to measure the period of rotation of an object from its light curve (Radigan 2014; Metchev et al. 2015 and references therein). Their periodic variability is caused by rotational modulation of the stellar flux generated by an asymmetric distribution of cool spots or spot groups on the stellar surface. These cool spots form mainly due to the presence of dust clouds. This can happen in various

*samrat687@gmail.com 
processes; the dust condensates are trapped in the atmosphere with permanent holes or low-density cloud formation in some places or a temporary formation of condensates and raining out causing quasi-periodic variation (Marley et al. 2010 and references therein). Irregular variations may happen due to highly variable, magnetically affected accretion from the circumstellar disk onto the star. The accretion rate is not only variable in time but the accretion zones may not be uniformly distributed over the stellar surface.

The reason for studying this class of objects in a star-forming region is that they offer their highest luminosity at a younger age. We monitored a specific region of IC348 with a pre-selected sample of BDs and M-dwarfs continuously in optical I band and in snapshot mode (in the optical R band). IC 348 is a young (2-3 Myrs) and nearby $(310 \mathrm{pc})$ star-forming region in the Perseus molecular cloud. It is a compact region and its membership and spectral types of members are well documented in the literature (Luhman et al. 2003; Luhman et al. 2005; Muench et al. 2007). A large population of low-mass stars and candidate brown dwarfs are found here. The paper is organized as follows: section 2 describes our observation log and data reduction process in brief. In section 3 , we have shown the phase curve of one of the sources and preliminary results are summarised in Table 2. We summarised our work in section 4.

\section{Observations and Data Reduction}

The photometric data were obtained using the 2-m Himalayan Chandra Telescope (HCT), Hanle operated by the Indian Institute of Astrophysics, Bangalore and 1.3-m Devasthal Fast Optical Telescope (DFOT), Devasthal operated by ARIES, Nainital. The details of the observations are given in Table 1.

Table 1: Observation log for IC 348.

\begin{tabular}{|c|c|c|c|c|c|}
\hline Date & Telescope & Instrument & $\begin{array}{l}\text { FoV } \\
\left(\operatorname{arcmin}^{2}\right)\end{array}$ & Exposure $(\mathrm{sec}) \times \mathrm{N}$ & $\begin{array}{l}\text { Run-Length } \\
\text { (hours) }\end{array}$ \\
\hline 18.12.2016 & 1.3-m DFOT & $\begin{array}{l}\text { ANDOR } \\
2 \mathrm{~K} \times 2 \mathrm{~K}\end{array}$ & $18 \times 18$ & $300 \times 1,400 \times 28$ & 3.77 \\
\hline 19.12.2016 & 1.3-m DFOT & $"$ & $"$ & $400 \times 19$ & 1.85 \\
\hline 20.12 .2016 & 1.3-m DFOT & $"$ & ” & $400 \times 40$ & 6.13 \\
\hline 27.10.2017 & 1.3-m DFOT & $"$ & ” & $250 \times 3,360 \times 1,300 \times 35$ & 6.99 \\
\hline 28.10.2017 & 1.3-m DFOT & $"$ & $"$ & $300 \times 29,360 \times 19$ & 4.64 \\
\hline 10.11.2017 & 1.3-m DFOT & $"$ & $"$ & $300 \times 45,360 \times 5$ & 6.16 \\
\hline 14.11.2017 & 2-m HCT & HFOSC & $10 \times 10$ & $300 \times 56$ & 7.98 \\
\hline 15.11.2017 & 2-m HCT & $"$ & $"$ & $300 \times 18,360 \times 43$ & 9.08 \\
\hline
\end{tabular}

\subsection{Data Analysis}

The raw CCD images were reduced using $\mathrm{IRAF}^{1}$ standard packages like bias subtraction, flat correction and cosmic ray removal. Then aperture photometry is done for the magnitude and flux estimation using the phot package of IRAF. We estimated the instrumental I-band magnitude for each source.

\footnotetext{
${ }^{1}$ Image Reduction and Analysis Facility (IRAF) is distributed by National Optical Astronomy Observatories (NOAO), USA (http://iraf.noao.edu/)
} 
Differential photometry was performed on all images to get a better precision for the light curves by removing the effects of atmospheric transparency and other instrumental signatures. In ensemble (differential) photometry, a sample of non-variable stars ( $\sim 20$ stars) is selected as the reference sample from the same frames as the targets with a comparable magnitude and an averaged light curve is created. The differential light curve of the target sources for each observing night is generated by subtracting the averaged light curves from that of the target sources, which removes all the artefacts due to the atmospheric and instrumental effects. The individual light curves of each source from all observing nights covering a range of 332 days in total from 2016 to 2017 (see Table 1) are then merged to get a final light curve for further period analysis.

\section{Results}

The candidate variables are selected from the magnitude vs. Root Mean Squared (RMS) magnitude of the individual light curve plot. The RMS of magnitudes measures the scatter in the light curve of an individual source. The RMS values of the light curves show a nearly exponential trend with the mean differential magnitude in CCD observations, where the candidate variables sit in the outliers from the normal trend. However, small-amplitude variables fall in or close to the normal trend, which requires visual inspection. Sometimes one or two points deviation due to improper correction of cosmic rays/position of the source at the CCD edge might show large RMS, which are also discarded after visual checking of the light curves. Here we searched for fast variability in low-mass stars and Brown Dwarfs with periods less than 1 day (Herbst et al. 2007; Biller 2017) from our dataset. The candidate variables list is submitted to a periodogram analysis and then to a visual inspection of the phase light curves. The Lomb-Scargle (LS) periodogram (Lomb 1976; Scargle 1982) is computed and the phase curve is constructed for the sources with the most significant periods. For example, Figure 1 represents the phase curve of the young M5 dwarf 2MASS J03443896+3203196, which is a member of IC 348 (Luhman et al. 2003). Some variable sources which present low-amplitude variations were selected by visual inspection. Some preliminary results for three variable candidates are shown in Table 2.

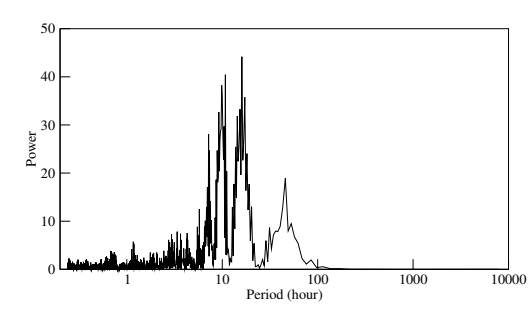

(a)

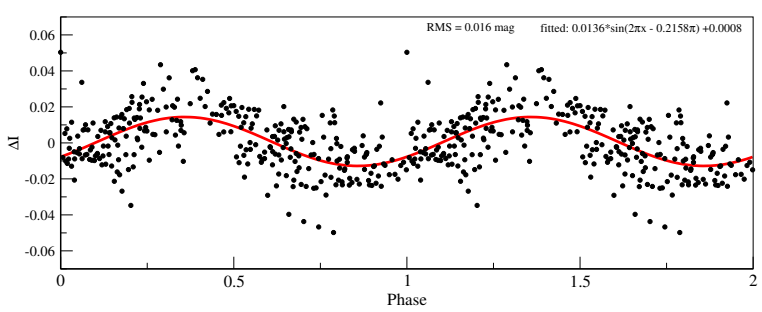

(b)

Figure 1: a. The Lomb-Scargle periodogram for the young low-mass star 2MASS J03443896+3203196 in IC 348 is shown here, which shows a significant period of $P=16.037$ hours. b. The phase light curve of that source using that period is shown here. We fitted a sine curve for better visual perception of the periodic nature.

\section{Summary}

From our observations, we find that 2MASS J03443896+3203196, a young M5 dwarf in IC 348, has clear periodic variability in our time-series I-band data with a period of 16.037 hours. Two other 
Table 2: Periods of few selected sources in IC 348 from our study.

\begin{tabular}{|c|c|c|c|c|c|c|}
\hline Star ID & Identifier & RA (h m s) & $\mathrm{DEC}(\operatorname{deg} \mathrm{m} \mathrm{s})$ & I mag & SpT & $\begin{array}{l}\text { Estimated } \\
\text { Period (hour) } \\
\text { (this work) }\end{array}$ \\
\hline 1 & $\begin{array}{l}\text { 2MASS J03445205 } \\
+3158252\end{array}$ & 034452.1 & +315825 & $16.86^{a}$ & $\mathrm{M} 3.5^{b}$ & 3.3 \\
\hline 4 & $\begin{array}{l}\text { [AMB2013]CFHT- } \\
\text { IC } 34817\end{array}$ & 034449.4 & +315844 & $17.91^{a}$ & & 4.58 \\
\hline 29 & $\begin{array}{l}\text { 2MASS J03443896 } \\
+3203196\end{array}$ & 034438.9 & +320319 & $17.24^{d}$ & $\mathrm{M} 5^{c}$ & 16.037 \\
\hline
\end{tabular}

${ }^{a}$ Monet et al. 2003; ${ }^{b}$ Muench et al. 2007; ${ }^{c}$ Luhman et al. 2003; ${ }^{d}$ Bell et al. 2013

sources show periodic variability from our preliminary analysis. We also found a few sources with large variations in magnitude in one or more nights but failed to determine any unique periodicity by LS periodogram analysis. These sources may either be aperiodic or have a longer period which is not included in our fast rotational analysis study.

\section{Acknowledgements}

This research work is supported by the S N Bose National Centre for Basic Sciences under the Department of Science and Technology, Govt. of India. The authors are thankful to the JTAC members and the staff of the 1.3-m Devasthal Fast Optical Telescope operated by the Aryabhatta Research Institute of Observational Sciences (ARIES, Nainital), the HTAC members and the staff of the HCT, operated by the Indian Institute of Astrophysics (Bangalore). SG is grateful to the Department of Science and Technology (DST), Govt. of India for their INSPIRE Fellowship scheme.

\section{References}

Allard F., Hauschildt P. H., Alexander D. R., Starrfield S. 1997, ARA\&A, 35, 137

Bell C. P. M., Naylor T., Mayne N. J., Jeffries R. D., Littlefair S. P. 2013, MNRAS, 434, 806

Biller B. 2017, AstRv, 13, 1

Crossfield I. J. M. 2014, A\&A, 566, A130

Herbst W., Maley J. A., Williams E. C. 2000, AJ, 120, 349

Herbst W., Eislöffel J., Mundt R., Scholz A. 2007, Protostars and Planets V, 297

Lomb N. R. 1976, Ap\&SS, 39, 447

Luhman K. L., Stauffer J. R., Muench A. A. et al. 2003, AJ, 593, 1093

Luhman K. L., Lada E. A., Muench A. A., Elston A. J. 2005, ApJ, 618, 810

Marley M. S., Saumon D., Goldblatt C. 2010, ApJ, 723, 117

Metchev S. A., Heinze A., Apai D. et al. 2015, ApJ, 799, 154

Monet D. G., Levine S. E., Canzian B., et al. 2003, AJ, 125,984

Muench A. A., Lada C. J., Luhman K. L., Muzerolle J., Young, E. 2007, AJ, 134, 411

Nakajima T., Oppenheimer B. R., Kulkarni S. R. et al. 1995, Natur, 378, 463

Radigan J. 2014, ApJ, 797, 120

Rockenfeller B., Bailer-Jones C. A. L., Mundt R. 2006, A\&A, 448, 1111

Scargle J. D. 1982, ApJ, 263, 835

Zapatero Osorio M. R., Martín E. L., Bouy H. et al. 2006, ApJ, 647, 1405 